\title{
effets non linéaires en dynamique des sols : essais in situ
}

\author{
non linear effects in soil dynamics in situ experiments
}

\author{
G. BONNET \\ J.-F. HEITZ \\ Institut de mécanique de Grenoble •
}

Rev. Franç. Géotech. n 46 , p.p. $19-30$ (janvier 1989)

\section{Résumé}

Devant le nombre croissant de codes de calcul d'ouvrages, un effort important est porté sur le développement d'essais de reconnaissance des sols in situ permettant de déterminer les paramètres dynamiques caractéristiques du comportement complexe des terrains de fondation. Dans cet article, l'essai de sismique harmonique de surface est présenté ainsi que le cheminement conduisant à interpréter au mieux cet essai. L'introduction d'une loi de comportenement non linéaire autorise une analyse harmonique de la réponse en accélération du sol sous sollicitations dynamiques. Un premier résultat fournit une estimation de la distorsion réalisée au cours de l'essai et montre la stabilité de la procédure de calcul.

\begin{abstract}
With the increasing number of computer programs for the simulation of foundations behaviour under seismic sollicitation, an important work is devoted to the development of in situ tests which allow to evaluate the parameters characterizing the dynamic behaviour of those foundations. In this paper, the surface harmonic seismic test and the progress leading to interpretate this test are presented. The introduction of non linear constitutive equations allows to analyse the harmonic spectrum of soil acceleration response under dynamic sollicitation. A first result gives an estimation of shear strain levels which occur during tests and shows the stability of the computation scheme.
\end{abstract}




\section{NOTATIONS}

$a_{n} \quad$ : fonction d'ordre $\mathrm{n}$ du second invariant du déviateur des déformations et du tenseur des déformations

$D_{n}^{m} \quad$ : coefficients de l'analyse de Fourier

$e_{i 1} \quad$ : tenseur des déformations

$\bar{e}_{i j} \quad$ : déviateur du tenseur des déformations

$e_{y} \quad$ : dilatation volumique

$F_{1} \quad$ : terme de source de non linéarités

$\bar{F}_{1} \quad$ : terme $F_{1}$ en approximation quasistatique

$\mathrm{G}_{\mathrm{n}} \quad$ : module de cisaillement d'ordre $\mathrm{n}$

$G \quad$ : module de cisaillement aux faibles distorsions

$\mathrm{l}_{2} \quad$ : second invariant du déviateur des déformations

L : opérateur différentiel linéaire

$u_{0}$ : déplacement du sol en approximation linéaire

$\mathrm{u}_{1} \quad$ : correction non linéaire du déplacement du sol

\section{INTRODUCTION}

Devant le nombre croissant de codes de calcul d'ouvrages en terre sous sollicitations dynamiques, il s'avère nécessaire de réaliser des essais permettant de déterminer expérimentalement in situ les caractéristiques non linéaires des sols sous de telles sollicitations.

Des essais ont été conduits par SHANNON et al. (SHANNON et al., 1980) mais sont difficiles à réaliser car ils nécessitent le forage de puits très proches et par ailleurs des énergies importantes. Le travail décrit ci-après s'inscrit dans une logique qui doit conduire à la réalisation d'essais nécessitant de faibles énergies : l'effort est porté sur linterprétation d'un effet secondaire de la réponse non linéaire qui est la modification du spectre entre Pexcitation et la réponse. En particulier, l'excitation, étant de type sinusoïdal établi, va générer une réponse non linéaire comprenant toutes les harmoniques de la fréquence du signal d'excitation. Ces harmoniques sont caractéristiques de la réponse non linéaire du sol.

\section{ESSAI DE SISMIQUE HARMONIQUE DE SURFACE (ESSAI S.H.S.)}

\subsection{Principe de l'essai}

L'essai se définit par la génération d'une excitation harmonique en surface et par la réception à distance de l'accélération du sol en surface (fig. 1). Cet essai génère préférentiellement des ondes de Rayleigh se propageant dans une zone proche de la surface. Ces ondes sont atténuées rapidement en profondeur.

Ce principe correspond exactement à l'essai classique d'ondes de Rayleigh stationnaire (RICHART et al., 1970) pour lequel il est proposé une utilisation et une interprétation originales dans le but de déterminer des paramètres caractéristiques du comportement non linéaire du sol. i. : accélération du sol

$\mathrm{u}_{i \mathrm{i}}^{*}$ : fonction de Green en déplacement

$\mathrm{X}$ : point de mesure du déplacement résultant

$\alpha, \alpha_{1}$ : paramètre caractéristique du comportement non linéaire

$\beta \quad$ : coefficient d'amortissement du sol

$\gamma \quad$ : distorsion

$\delta \quad$ : symbole de Kronecker

$\epsilon \quad$ : petit paramètre

$\lambda_{n} \quad$ : coefficient de Lamé d'ordre $n$

$\lambda_{c} \quad$ : coefficient de Lamé aux faibles distorsions

$\nu \quad$ : coefficient de Poisson

$\rho \quad$ : densité volumique du sol

$\sigma_{i j} \quad$ : tenseur des contraintes

$\xi$ : point d'application de la force correspondant à $\mathrm{F}_{1}$

$\Omega \quad$ : domaine des non linéarités

\subsection{Description de l'essai}

Une source harmonique est utilisée à l'excitation. Cette source est constituée d'un piston couplé à un pot vibrant (fig. 2) et pilotée par le générateur d'un analyseur de réponse en fréquence type SOLARTRON 1250 (EnertecSchlumberger) au moyen duquel on fixe la fréquence d'excitation. Un amplificateur de puissance fournit lamplitude de la force dynamique désirée. Cette force est contrôlée par un capteur de force dynamique (piézoélectrique). Pour assurer un bon couplage piston-sol, une charge statique, contrôlée par un capteur de force statique, est appliquée directement sur le piston par vérin pneumatique.

A la réception, deux accéléromètres (composante verticale) sont placés à la source et à distance. De plus, pour connaitre la réponse du sol suivant les composantes radiale et verticale de façon simultanée, un vélocimètre est disponible pour la mesure. La réponse en accélération spectrale est obtenue grâce à l'analyseur qui permet d'obtenir le spectre d'harmoniques relatif à chacune de ces mesures.

Cet essai nécessite également des conditionneurs (ou amplificateurs de charge) associés à chacun des capteurs ainsi qu'un oscilloscope permettant de contrôler à tout moment la forme des signaux d'excitation et de réception. Un schéma du montage de l'essai est présenté sur la figure 3.

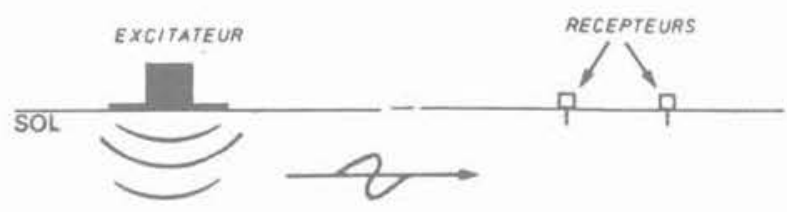

Fig. 1. - Principe de I'essai S.H.S.

Fig. 1 - Principle of S.H.S. experiment. 


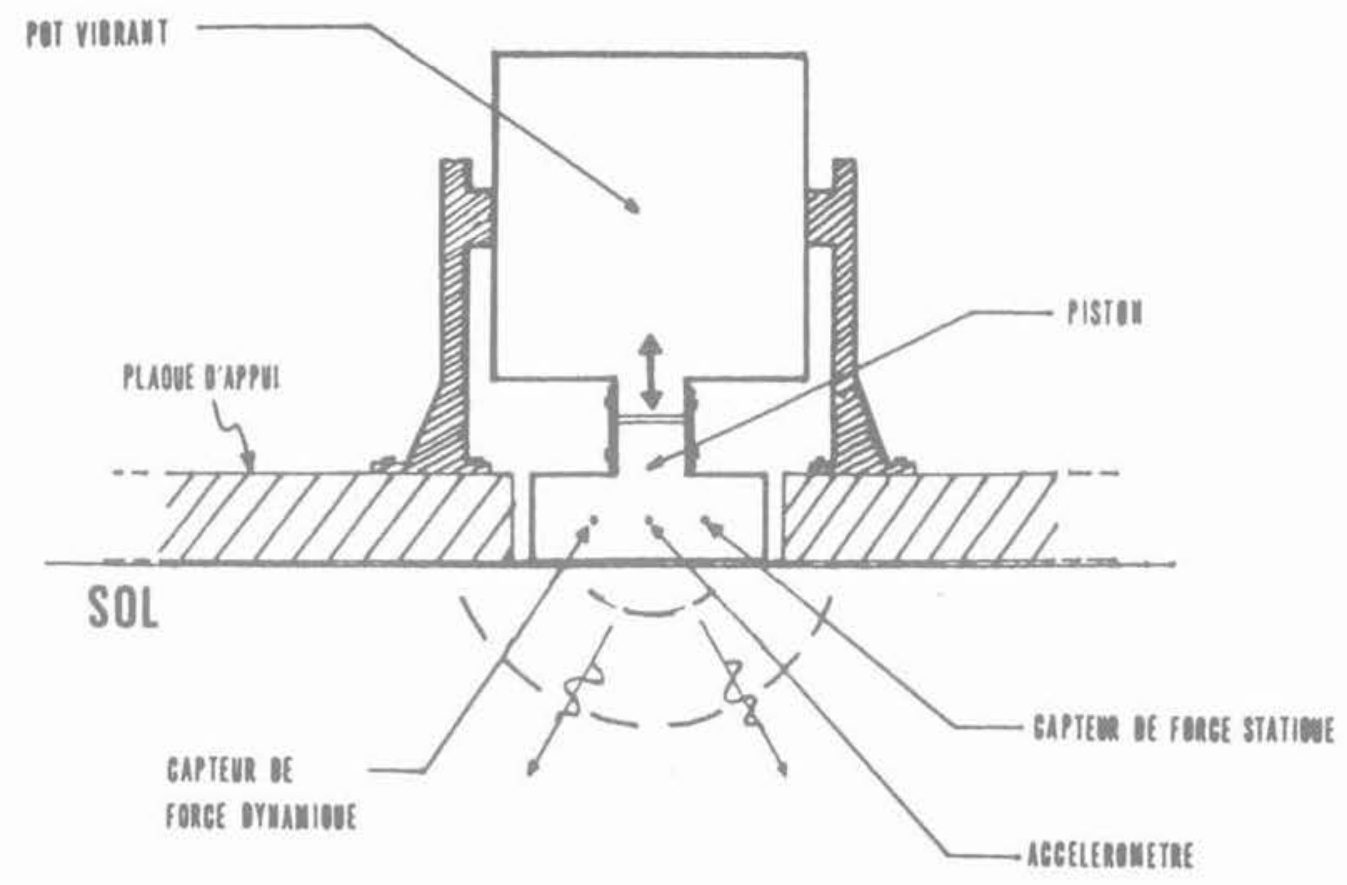

Fig. 2. - Tête d'excitation de l'essai S.H.S.

Fig. 2. - Loading part of S.H.S. experiment.

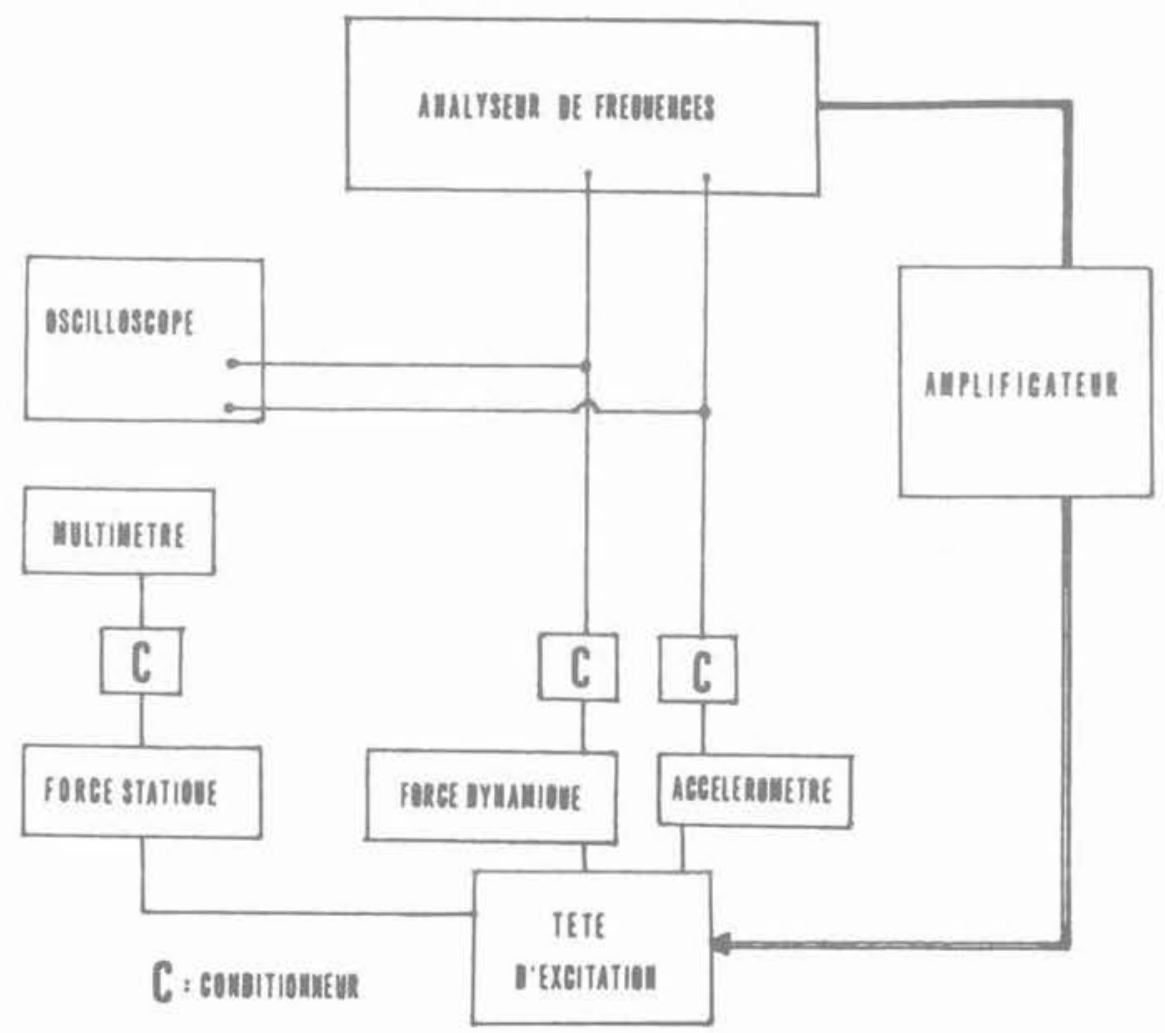

Fig. 3. - Chaîne instrumentale.

Fig. 3. - Experimental set-up. 


\subsection{Intérêt de l'essai}

L'utilisation d'une source sismique harmonique présente lavantage de pouvoir contrôler le contenu fréquentiel de l'excitation. Cet essai peut donc être mis en œuvre dans un environnement bruyant pourvu que le contenu spectral du bruit n'interfère pas avec le spectre du signal utile. Ceci garantit à la réception un enregistrement à bon rapport signal sur bruit.

Par ailleurs, l'émission d'un signal monofréquentiel (sinusoïdal) permet d'obtenir à la réception directement la fonction de transfert du sol sans traitement particulier et de ne mesurer finalement que des amplitudes et des phases.

Enfin, le caractère non destructif de l'essai autorise la reproduction des mesures.

\subsection{Déroulement des essais}

Un premier essai à faible niveau de distorsion $\left(\gamma<10^{-5}\right)$ permet à partir de la mesure des amplitudes et des phases d'évaluer les modules de cisaillement $G$ et d'amortissement $\beta$ du sol.

Les essais ultérieurs, à niveau croissant de distorsion, permettent d'obtenir les spectres de réponse en accélération à partir desquels les paramètres dynamiques non linéaires sont calculés.

\subsection{Résultats expérimentaux}

La figure 4 montre les résultats obtenus en surface d'un sol composé de limon argileux. On peut constater que le spectre d'accélération A comprend des harmoniques dont l'amplitude peut aller jusqu'à $80 \%$ de l'amplitude obtenue à la fréquence fondamentale et ceci bien que la force d'excitation $F$ soit parfaitement sinusoïdale, comme le montre le spectre de $\mathrm{F}$ reporté en figure 4. C'est la génération de ces harmoniques qu'il faut interpréter pour remonter aux caractéristiques du comportement non linéaire du sol. Cette interprétation fait l'objet des paragraphes suivants.

\section{MODÉLISATION DU COMPORTEMENT NON LINÉAIRE DES SOLS}

Des essais dynamiques au laboratoire ont permis à SEED et al. de tracer des courbes moyennes expérimentales de l'évolution du module de cisaillement $G$ et du coefficient d'amortissement $\beta$ en fonction du niveau de distorsion $\gamma$ pour des sables pulvérulents et des argiles (SEED et al., 1973).

Ces résultats montrent que la rigidité décroît et que lamortissement croit lorsque le niveau de distorsion augmente (fig. 5).

De nombreux auteurs ont proposé de formaliser ce comportement particulier : HARDIN et DRNEVICH, 1970 ; RAMBERG et OSGOOD (RICHART et al., 1975) mais le formalisme proposé est construit en configuration unidimensionnelle.

On se propose ci-après de généraliser ce comportement en configuration tridimensionnelle.

\subsection{Choix de la variable}

Pour obtenir une écriture indépendante du référentiel choisi, nous proposons d'exprimer que les coefficients élastiques ne dépendent que du second invariant du déviateur des déformations, noté $1_{2}$, dont l'expression est donnée ci-après :

$$
I_{2}=\sum_{\substack{n, j \\ i \neq 1}}\left(\tilde{e}_{i j} \cdot \tilde{e}_{i j}-\tilde{e}_{i j}\right)
$$

avec $\tilde{e}_{i}$ qui représente le déviateur des déformations et qui s'exprime en fonction du tenseur $e_{i j}$ des déformations et de la dilatation volumique $e_{y}$, tel que :

$$
\bar{e}_{i j}=e_{i j}-e_{v} / 3 \cdot \delta_{i j}
$$

où $\delta_{i j}$ est le symbole de Kronecker tel que $\delta_{i j}=1$ pour $i=j$ et $\delta_{i 1}=0$ pour $i \neq j$.

Dans le cas particulier unidimensionnel de l'excitation d'un horizon de sol par une contrainte de cisaillement à sa base, seule la composante $e_{x z}$ du tenseur des déformations est non nulle.

De ce fait, $I_{2}$ devient :

$$
I_{2}=-1 / 4 \cdot \gamma^{2} \text { où } \gamma=2 e_{x 2}
$$

On retrouve alors le schéma classique où $G$ n'est fonction que de $\gamma$. Si nous admettons que la réponse du sol est identique en traction et compression, il est nécessaire d'utiliser dans le formalisme, le second invariant $I_{2}$ en module soit $\left|I_{2}\right|$.

\subsection{Choix de la loi de comportement adoptée}

La loi de Hardin-Drnevich (1970) stipule que $\mathrm{G} / \mathrm{G}_{0}=$ $1 /(1+\alpha|\gamma|)$. On peut envisager une généralisation de cette loi telle que:

$$
\mathrm{G} / \mathrm{G}_{\mathrm{o}}\left(\mathrm{I}_{2}\right)=1 /\left(1+\alpha \cdot\left|\mathrm{I}_{2}\right|^{1 / 2}\right)
$$

Toutefois, ce type de loi présente l'inconvénient suivant : lorsque l'on étudie la réponse spectrale du sol, il faut calculer le spectre d'une fonction de $G / G_{0}$ (cf. ciaprès). Avec une loi du type ci-dessus, il n'est pas possible de calculer analytiquement le spectre de la réponse, sur lequel repose toute l'interprétation.

On peut envisager d'utiliser le développement en série de l'expression (2.2.1.) mais ce développement a un rayon de convergence trop faible. Il est donc nécessaire d'utiliser une expression différente de $G / G_{0}$, pour laquelle le rayon de convergence soit plus important. 

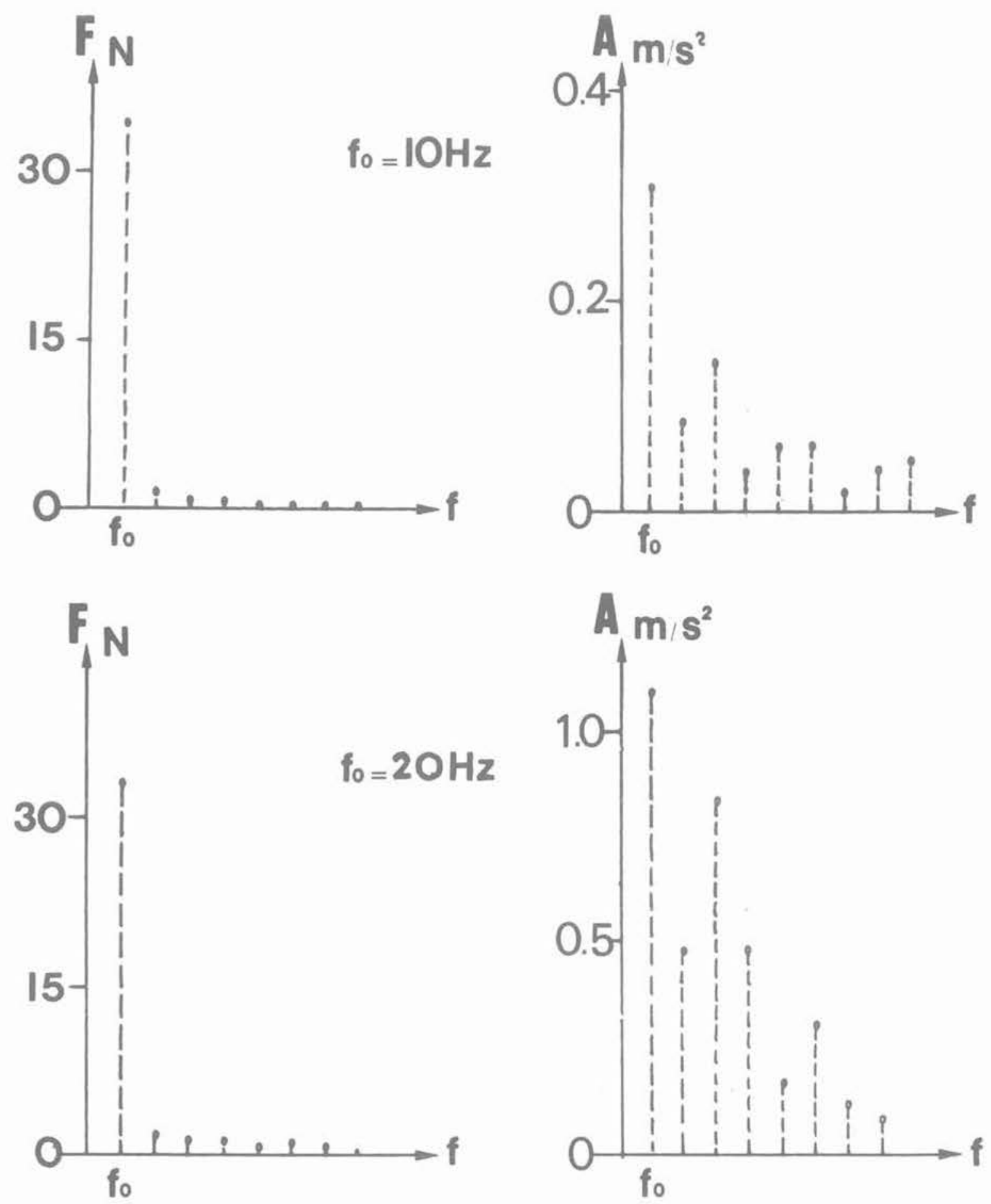

Fig. 4. - Résultats expérimentaux.

Fig. 4. - Experimental results. 
a) Sols pulvêrulents
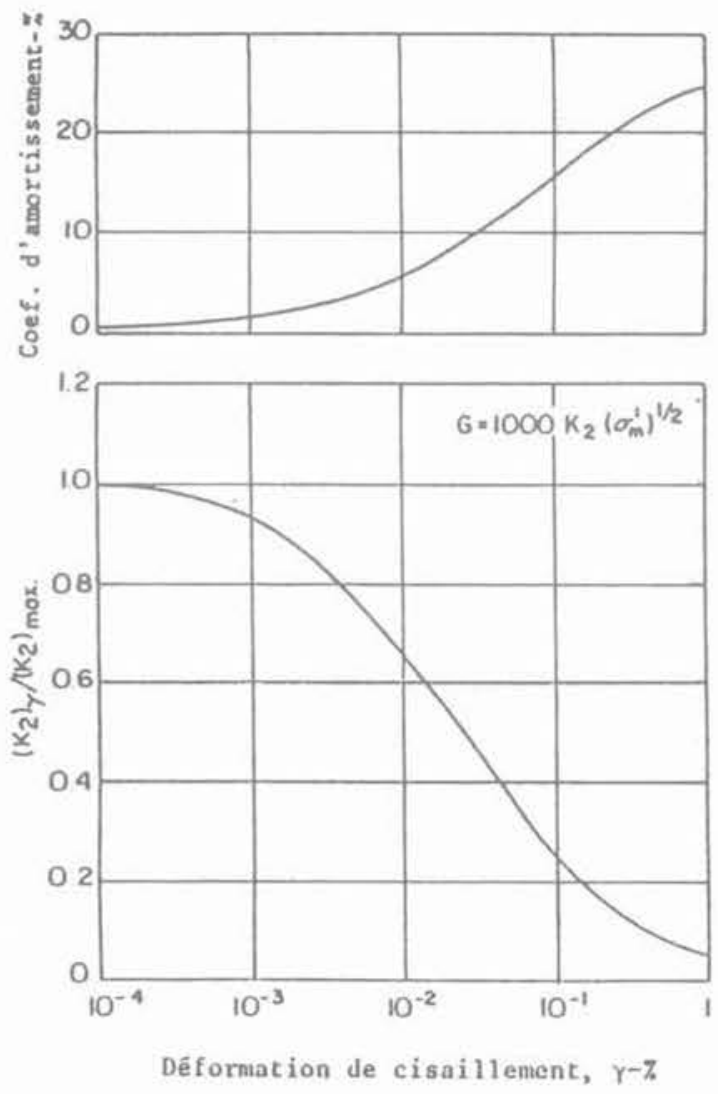

b) Argilıs saturếes
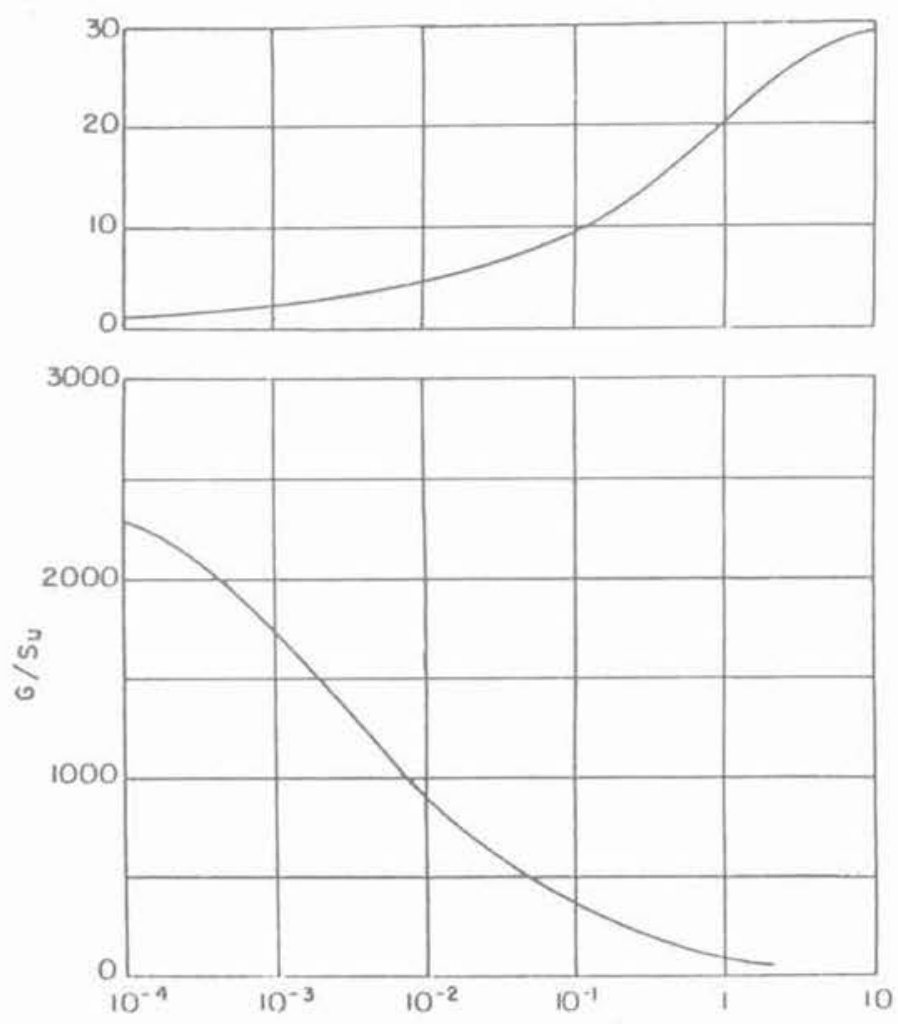

Dêformation de cisaillement, $\gamma-\%$

Fig. 5. - Courbes moyennes de SEED (d'après SEED et al., 1973).

Fig 5. - Seed's mean curves lafter SEED et al., 1973)

Notre choix s'est porté sur une fonction de type exponentiel :

$$
\mathrm{G} / \mathrm{G}_{0}\left(\mathrm{I}_{2}\right)=\exp \left(-\alpha_{1} \cdot\left|I_{2}\right|^{1 / 2}\right)
$$

qui présente un rayon de convergence infini en $\mathrm{I}_{2}$ et dont le développement en série s'écrit :

$$
\mathrm{G} / \mathrm{G}_{0}\left(\mathrm{I}_{2}\right)=1+\sum_{n=1}^{\infty}(-1)^{n} \frac{\alpha_{1}^{n}}{n !}\left|\mathrm{I}_{2}\right|^{n / 2}
$$

La figure 6 montre la courbe $G / G$ o obtenue que l'on peut comparer aux différentes expressions classiques. La courbe obtenue est bien placée pour représenter les résultats expérimentaux. Cette expression sera donc utilisée dans la suite pour l'interprétation des essais.

\section{INTERPRÉTATION DE L'ESSAI S.H.S}

L'interprétation repose essentiellement sur deux points : - calcul du spectre d'harmoniques générées au cours de l'essai :
- utilisation d'une méthode de perturbation à partir de la réponse linéaire.

4.1. Calcul du champ d'ondes généré au cours de l'essai : principe du calcul

\subsubsection{Equations du problème}

Soit $\sigma_{i j}$ le tenseur des contraintes développées dans le sol. Si $\rho$ est la densité du sol et $\ddot{u}_{i}$, le champ d'accélération dans le sol dérivé du champ de déplacement $u_{i}$. la loi de la dynamique s'écrit :

$$
\sigma_{i j . j}=\rho \ddot{u}_{i}
$$

Le comportement adopté pour le sol s'exprime:

$$
\sigma_{i j}=\lambda\left(I_{2}\right) e_{v} \delta_{i j}+2 G\left(I_{2}\right) e_{i j}
$$

avec

$$
\lambda\left(I_{2}\right)=\lambda_{0}+\sum_{n=1}^{\infty}\left((-1)^{n} / n !\right) \lambda_{n} \cdot\left|I_{2}\right|^{n / 2}
$$




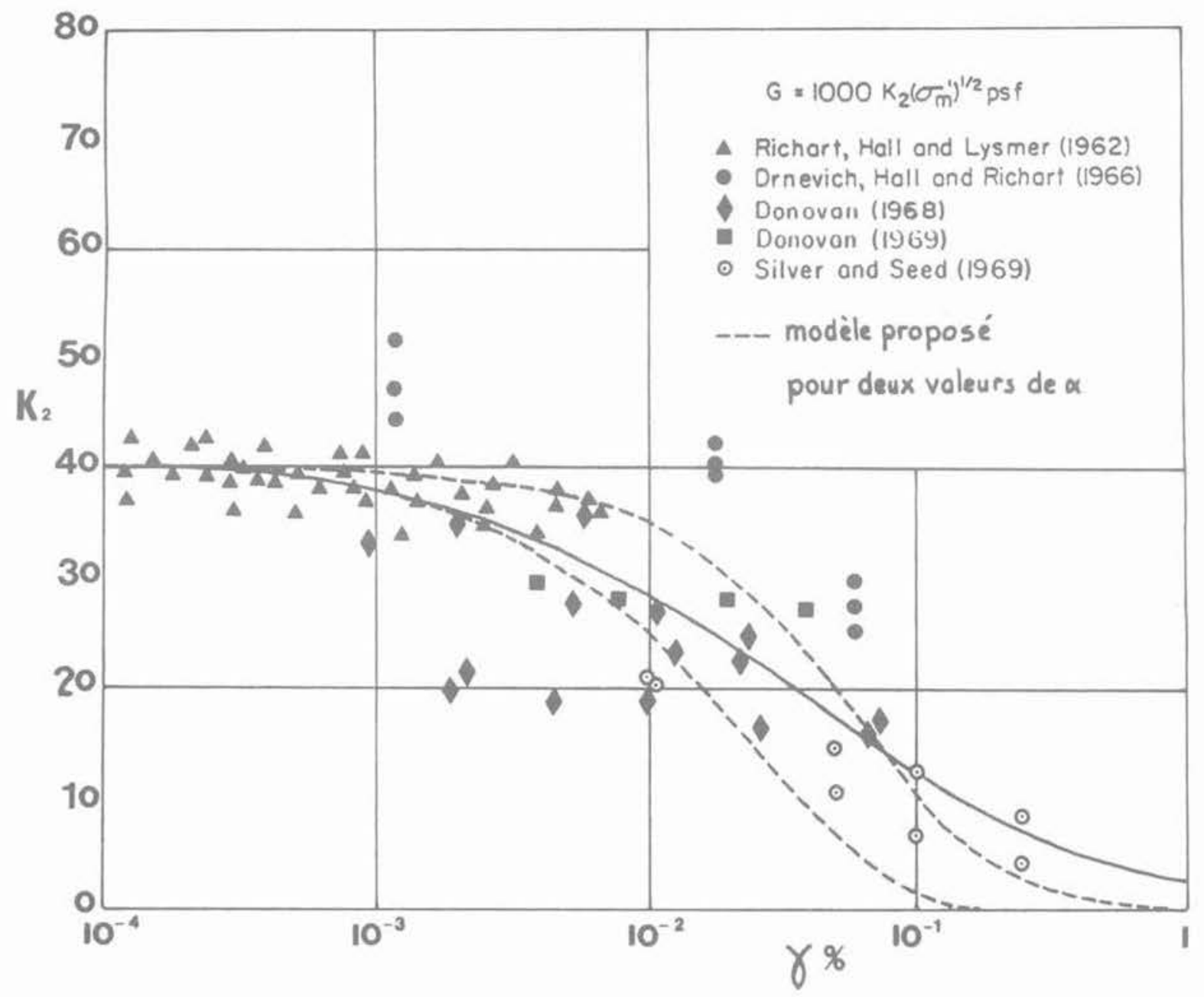

Fig. 6. - Résultats expérimentaux (d'après SEED et al., 1973) et modélisation proposée de la variation

du madule de cisaillement $G$ en fonction de la distorsion.

$G\left(I_{2}\right)=G_{0}+\sum_{n=1}^{\infty}\left((-1)^{n} / n !\right) G_{n} \cdot\left|I_{2}\right|^{n / 2}$

si : $\quad \lambda_{n}=b(\nu) \cdot G_{n}$ et $G_{n}=\alpha_{1}^{n} \cdot G_{o}$

alors: $\lambda_{\mathrm{n}}=\mathrm{b}(\nu) \mathrm{G}_{0} \alpha_{1}{ }^{n}$

mais: $\lambda_{0}=b(\nu) \cdot G_{0}$ d'où : $\lambda_{\mathrm{n}}=\alpha_{1}^{n} \cdot \lambda_{0}$

L'équation résultant des expressions (3.1.1) et (3.1.2) permettant le calcul du déplacement $u_{i}$ est :

$$
\left(\lambda\left(I_{2}\right) e_{v} \delta_{i j}+2 G\left(I_{2}\right) e_{i j}\right)_{+j}-\rho \ddot{u}_{i}=0 \quad(3.1 .5)
$$

Le développement de cette équation nécessite de connaitre les expressions $\left(\lambda\left(I_{2}\right)\right)$, et $\left(G\left(I_{2}\right)\right)$, décrites ci-après :
Fig. 6. - Experimental results fatter SEED et al, 1973) and proposed modelization of shear strain madulus $G$ variation interns of shear strain level
$\left(\left(\lambda\left(I_{2}\right)\right)_{, 1}=\sum_{n=1}^{\infty}\left((-1)^{n} \lambda_{n} / 2(n-1) !\right) \cdot\left|I_{2}\right|^{n / 2-1} \cdot\left|I_{2}\right|_{n}\right.$

$\left(\left(G\left(I_{2}\right)\right)_{y}=\sum_{n=1}^{\infty}\left((-1)^{n} G_{n} / 2(n-1) !\right) \cdot\left|I_{2}\right|^{n / 2-1} \cdot\left|I_{2}\right|\right.$,

Etant donné que : $e_{i j}=\left(u_{i, j}+u_{i, j}\right) / 2$

L'équation du problème devient :

$$
\left(\lambda_{0}+G_{o}\right) u_{i, j i}+G_{0} u_{i, j l}-\rho \ddot{u}_{i}=F_{i}
$$


où l’on a posé :

$$
\begin{aligned}
& F_{1}=\left(\sum_{n=1}^{\infty}\left((-1)^{n+1} / n !\right) \cdot\left|I_{2}\right|^{n / 2}\left(\lambda_{n}+G_{n}\right) u_{j, i j}\right. \\
& +\left(\sum_{n=1}^{\infty}\left((-1)^{n+1} / n !\right) \cdot\left|I_{2}\right|^{n / 2} G_{n}\right) u_{i, j} \\
& +\left(\sum _ { n = 1 } ^ { \infty } ( ( - 1 ) ^ { n + 1 } / 2 ( n - 1 ) ! ) \cdot | I _ { 2 } | ^ { n / 2 - 1 } \left(\lambda_{n}\left|I_{2}\right|, e_{v}\right.\right. \\
& \left.+2 G_{n}\left|I_{2}\right|, e_{j}\right)
\end{aligned}
$$

\subsubsection{Méthode de résolution}

La méthode de résolution adoptée pour calculer $u_{1}$ est une méthode de perturbation qui repose sur l'hypothèse suivante: le terme $F_{1}$ du second membre est petit devant chacun des termes du premier membre.

L'équation (3.1.9) peut s'écrir :

$$
\mathrm{L}\left(\mathrm{u}_{1}\right)=\mathrm{F}\left(\mathrm{u}_{i}\right)=\epsilon \mathrm{F}^{\prime}\left(\mathrm{u}_{i}\right)
$$

où $€$ est petit devant l'unité et $\mathrm{L}$, un opérateur différentiel linéaire apparaissant dans le premier membre de l'équation. $\mathrm{F}\left(\mathrm{u}_{\mathrm{i}}\right)$ a pour composante $\mathrm{F}_{\mathrm{i}}$.

$\epsilon$ étant petit, on peut s'attendre à ce que la solution $u_{1}$ de (3.1.11) soit proche de la solution $U_{o_{1}}$ telle que :

$$
\mathrm{L}\left(\mathrm{U}_{0_{1}}\right)=0
$$

$\mathrm{u}_{0}$ est appelée solution génératrice (BLAQUIÈRE, 1966) La solution de l'équation (3.1.11) sécrit alors : $\mathrm{u}=\mathrm{u}_{0}+$ $\mathrm{u}_{1}$, où $\mathrm{u}_{1}$ est petit devant $\mathrm{u}_{0}$. On doit donc vérifier :

$$
L\left(u_{0}+u_{1}\right)=F\left(u_{0}+u_{1}\right)
$$

Puisque $u_{1}$ est petit devant $u_{0}$, on peut poser $u_{1}=\varepsilon u_{1}{ }^{\circ}$, où $u_{1}{ }^{*}$ est du même ordre que $u_{0}$. En remplaçant dans l'équation ci-dessous, on obtient :

$$
\epsilon L\left(u_{1}^{*}\right)=F\left(u_{0}+\epsilon u_{1}^{*}\right) \simeq \epsilon F^{*}\left(u_{0}\right)
$$

La correction $\mathrm{u}_{1}$ est donc solution de :

$$
\mathrm{L}\left(\mathrm{u}_{1}\right)=\mathrm{F}\left(\mathrm{u}_{0}\right)
$$

La résolution de l'équation (3.1.15) se décompose donc en deux étapes :

a. obtention de lapproximation linéraire $\mathrm{u}_{0}$, solution de : $\mathrm{L}\left(\mathrm{u}_{0}\right)=0$

b. calcul de la correction $u_{1}$, solution de : $L\left(u_{1}=F\left(u_{0}\right)\right.$.

La méthode exposée est tout à fait générale et peut être simplifiée sous l'hypothèse suivante : le terme $F_{1}$ apparaissant au second membre de (3.1.9) n'est à prendre en compte qu'au voisinage de la source dans un domaine $\Omega$ où les distorsions sont importantes. Dans ce cas, si l'extension de ce domaine de non linéarités $\Omega$ est faible devant la longueur d'onde, on peut supposer que la réponse du sol en approximation linéaire est en phase avec la sollicita- tion. Cette hypothèse revient donc à considérer que le champ peut être calculé à partir d'une approximation \& quasi-statique » où les termes d'inertie peuvent être négligés. L'équation à résoudre s'écrit alors :

$$
\left(\lambda_{0}+G_{0}\right) u_{j, i j}+G_{0} u_{i j i j}=\tilde{F}_{j}
$$

où les $\mathrm{F}_{\text {, }}$ sont calculés à partir de l'approximation quasi-statique.

\subsubsection{Sources de non linéarités}

Les sources de non linarités $\mathrm{F}_{1}$ se calculent à partir de $\mathrm{I}_{2}$ et $e_{i j}$. Par ailleurs si $u_{0}$ est le champ en approximation linéaire, on a :

$u_{0_{1}}=\bar{u}_{0} \cdot \cos w t, e_{i j}=\overline{e_{i j}} \cdot \cos w t$ et $I_{2}=\bar{I}_{2} \cdot \cos ^{2} w t$. On obtient ainsi :

$\left.\bar{F}_{1}=\sum_{n=1}^{\infty} a_{n} \overline{\left(I_{2}\right.}, \overline{e_{i j}}\right) \cdot\left|\cos ^{2} w t\right|^{n / 2} \cdot \cos w t$

avec :

$a_{n}=\left.\left((-1)^{n+1} / 2(n-1) !\right) \cdot \bar{T}_{2}\right|^{n / 2-1}\left[\lambda_{n} \bar{I}_{2} \mid, 1, \overline{u_{j, j}}\right.$

$\left.+2 G_{n} \Pi_{2} \mid, j \cdot \bar{e}_{j j}\right]$

On suppose que $\nu$ est constant, il existe alors une relation simple entre

$\left.a_{n+1} \overline{(I}_{2}, \bar{e}_{i j}\right)$ et $a_{n}\left(\bar{I}_{2}, \bar{e}_{i j}\right)$ telle que :

$$
a_{n+1}=\left.\left(-\alpha_{1} / n\right) \cdot \pi_{2}\right|^{1 / 2} a_{n}
$$

Cette formule de récurrence permet de calculer tous les $a_{n}$ en fonction de $a_{1}$ :

$$
a_{n}=\left(\left(-\left.\alpha_{1} \cdot \bar{I}_{2}\right|^{1 / 2}\right)^{n-1} /(n-1) !\right) \cdot a_{1}
$$

avec:

$a_{1}=\left.G_{1} \cdot \bar{I}_{2}\right|^{-1 / 2} \cdot\left[b(v) / 2 \cdot \bar{I}_{2}\left|, i \cdot \overline{u_{j, j}}+\right| \bar{I}_{2} \mid, j \cdot \bar{e}_{1}\right]$

Considérons maintenant la partie temporelle de $\mathrm{I}_{2}$. On remarque immédiatement :

$\left|\cos ^{2} w t\right|^{n / 2} \cdot \cos w t=$

$|\cos w t|^{n} \cdot \cos w t=\sum_{n=1}^{\infty} D_{n}^{m} \cdot \cos (2 m-1) w t$

Les coefficients $D_{n}^{m}$ sont obtenus par analyse en série de Fourier. Leur calcul montre que :

- si $m=2 q, D_{n}^{m}=0, \forall_{n}$

- si $\mathrm{m}=2 \mathrm{q}-1$,

et $\mathrm{n}=2 \mathrm{p}$,

D ${ }_{2 \mathrm{p}}^{2 \mathrm{q}-1}=((2 \mathrm{p}-1) ! /((\mathrm{p}-\mathrm{q}) !(\mathrm{p}+\mathrm{q}-1) !)) / 2^{\mathrm{p}-1}$ et $\mathrm{n}=2 \mathrm{p}-1$,

D $\frac{2 q-1}{2 p-1}=\sum_{s=1}^{p} D_{2(p-1)}^{S} \cdot K_{2 s-1}^{2 q-1}$ 
avec :

$K \begin{aligned} & 2 q-1 \\ & 2 s-1\end{aligned}=$

$\frac{(-1)^{s+a+2}}{\pi} \cdot \frac{8 m(2 s-1)}{\left[m_{2}-4(s-1)^{2}\right]\left[m^{2}-4 s^{2}\right]}$

Finalement Pexpression complète du terme $F_{1}$, source de non linéarités, est donnée par:

$$
\begin{aligned}
\tilde{F}_{1} & =a_{1} \cdot \sum_{m=1}^{\infty}\left\{\sum_{n=1}^{\infty}(-\alpha)^{n-1} /(n-1) !\right) \\
& \left.\left.\cdot T_{2}\right|^{(n-1) / 2}, D_{n}^{m}\right] \cdot \operatorname{Cos}(2 m-1) w t
\end{aligned}
$$

Le spectre de force correspondant est un spectre d'harmoniques impaires. C'est l'hypothèse d'une réponse identique du sol en traction et en compression qui conduit à ce résultat.

\subsubsection{Calcul de la correction non linéaire}

La correction non linéaire $\mathrm{U}_{1}$ est solution de (3.1.15). La solution s'écrit alors :

$$
u_{11}(x)=\int_{0 \xi} u_{i j}^{*}(x ; \xi) \cdot F_{j}(\xi) d \Omega_{\xi}
$$

où $u_{i}^{*}$ est la fonction de Green correspondant à un demi-espace et se définit comme le déplacement élémentaire au point $\mathrm{X}$, dans la direction $\mathrm{i}$, créé par une force unité sur un anneau passant par le point $\xi$, dans la direction j (fig. 7). $\Omega_{\xi}$ correspond au domaine sur lequel les distorsions sont suffisamment importantes pour que les termes non linéaires interviennent.

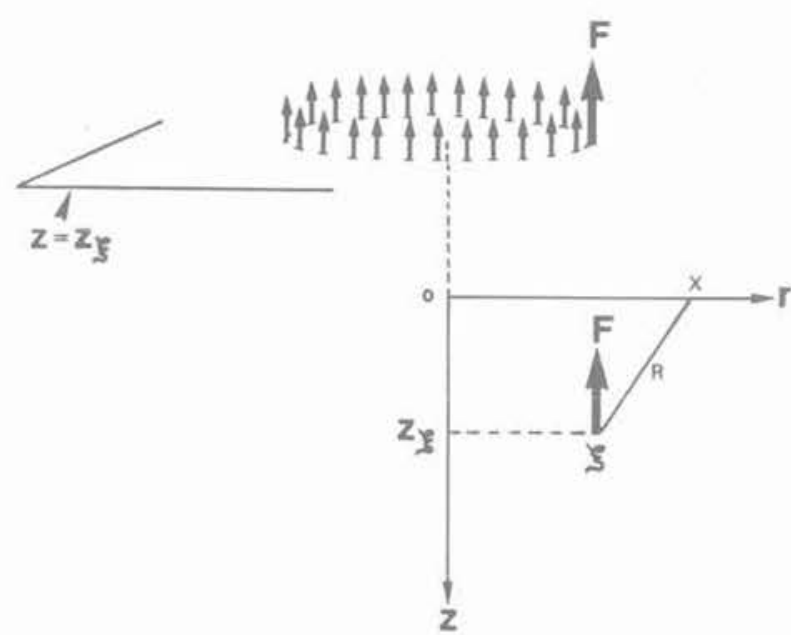

Fig. 7. - Force annulaire équivalente en représentation axisymétrique.

Fig. 7. - Force equivalent to a circular distribution with axisymmetry.

Notons que $u_{i j}^{*}$ s'exprime sur la base des intégrales elliptiques complètes de $1_{e}, 2_{e}$ et 3 espèces et que les composantes du déplacement $\mathrm{u}_{\mathrm{i}}$ considérées ci-dessus permettent de déterminer l'amplitude d'une des composantes du spectre. Les autres composantes du spectre sont obtenues comme décrit au paragraphe 3.1.4.
En résumé, le calcul comprend quatre phases :

a. calcul de l'approximation linéaire (3.1.12) ;

b. calcul des termes de sources (3.1.22) ;

c. calcul des fonctions de Green, $u_{i j}^{*}$;

d. calcul de la correction non linéaire (3.1.15).

\subsection{Différentes phases du calcul}

a. Calcul de l'approximation linéaire.

Le calcul de l'approximation linéaire dans l'hypothèse quasi-statique est fourni par les solutions de GERARD et HARRISSON rappelées dans l'ouvrage classique de DAVIS et POULOS 1974 dont on donne en annexe le détail.

b. Calcul des termes de source

Les termes de source sont obtenus en dérivant le déplacement $\mathrm{u}_{0}$ en approximation quasi-statique. Le programme de calcul symbolique * REDUCE » a été utilisé pour effectuer ces dérivations.

c. Calcul des fonctions de Green $u_{i j}^{*}$ en représentation axisymétrique.

Ces fonctions de Green sont obtenues en intégrant les solutions classiques correspondant au demi-espace (BREBBIA et al., 1984).

d. Calcul de la correction non linéaire

La convolution correspondant à l'intégrale (3.1.23) est calculée numériquement en décomposant le volume d'intégration, rapporté à un domaine bidimensionnel par

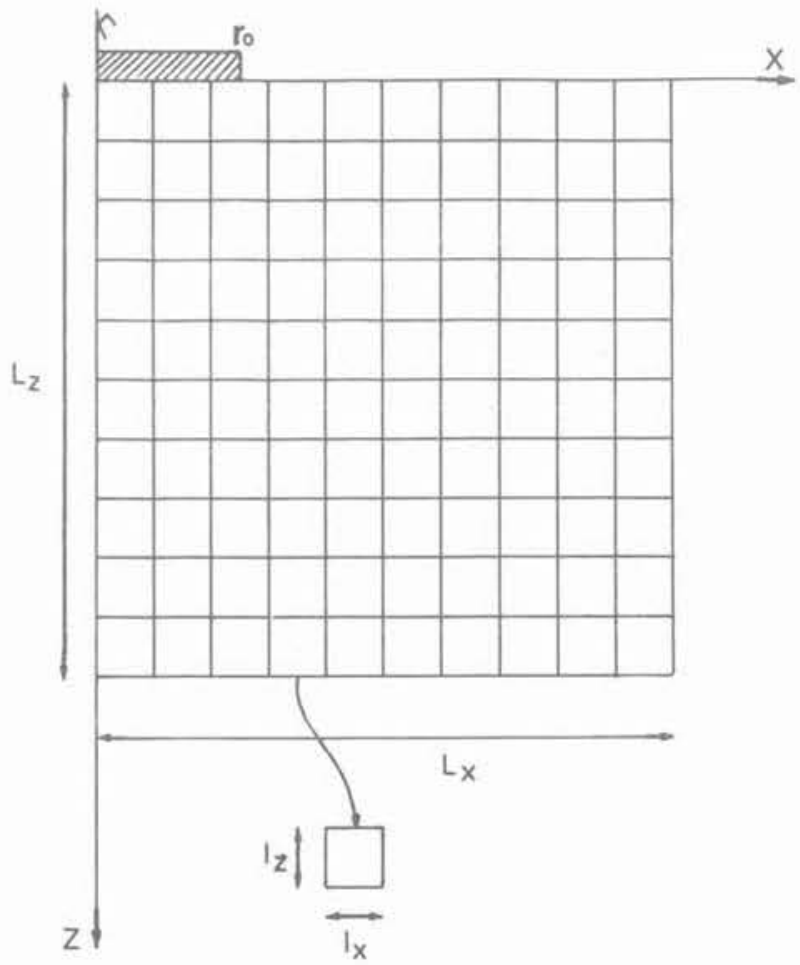

Fig. 8. - Maillage du domaine des non linéarités. Fig. 8. - Mesh of the non linear domain. 
propriété d'axisymétrie, sur un ensemble d'éléments rectangulaires (fig. 8). C'est la seule phase numérique du calcul.

\subsection{Programme de calcul}

Le programme de calcul utilisant la méthode décrite précédemment a été mis au point. L'intégration numérique nécessaire au calcul de l'intégrale $(3,1.23)$ s'effectue par quadrature de Gauss, excepté pour l'élément tel que X $=\xi$ qui nécessite une intégration singulière. Celle-ci est réalisée au moyen de la méthode de MANG (MANG et al., 1985) qui consiste essentiellement en une réduction de lordre de singularité en $1 / \mathrm{r}$ par transformation géométrique.

\section{PREMIERS RESULTATS NUMERIQUES}

\subsection{Calcul du champ des distorsions}

On a effectué, pour le maillage reporté en figure 8 , le calcul du champ des distorsions $\left|\mathrm{I}_{2}\right|^{1 / 2}$ correspondant à lessai décrit précédemment. Les résultat sont reportés en figure 9. Bien entendu, la distorsion diverge au voisinage du bord du piston excitateur mais le calcul donne la valeur de la distorsion moyenne sur l'élément corres. pondant. On peut constater que la distorsion moyenne maximale obtenue est de l'ordre de $10^{-4}$. Cela permet de déterminer le nombre de termes à conserver dans le développement de $\mathrm{G} / \mathrm{G}_{\mathrm{o}}(2.2 .4$.) pour le calcul des termes de source.

\subsection{Convergence du processus d'intégration}

Un calcul de la correction non linéaire $u_{1}$ a été effectué pour une distorsion moyenne $\gamma=10^{-5}$ pour laquelle on peut conserver un seul terme du développement, soit :

$$
\mathrm{G} / \mathrm{G}_{\mathrm{o}}\left(\mathrm{I}_{2}\right)=\exp \left(-\alpha_{1}\left|I_{2}\right|^{1 / 2}\right)=1-\alpha_{1}\left|\mathrm{I}_{2}\right|^{1 / 2}
$$

Ce calcul a été effectué pour plusieurs types de maillage et plusieurs extensions de la zone d'intégration des termes non linéaires. Les tableaux 1 et 2 et la figure 10 permettent de constater que la convergence est assurée.

\section{CONCLUSION}

L'essai S.H.S. réalisé permet d'assurer des distorsions importantes. L'interprétation développée ci-dessus a permis de montrer que l'existence de telles distorsions conduit à un spectre de réponse comprenant toutes les harmoniques impaires de la fréquence du signal d'excitation, Par ailleurs cette interprétation permet d'obtenir les distorsions réalisées au cours de l'essai.

Le calcul effectué au § 5.2. qui a permis de conclure à la convergence du processus d'intégration ne permet
Tableau 1. - Déplacement vertical calculé fonction de I'extension $L$ du maillage $\left(I x / r_{0}=0.4\right.$ et $\left./ z / r_{0}=0.5\right)$ Table 1. - Computed vertical displacement in terms of $L$ extent $\left(I x / r_{0}=0.4\right.$ and $\left.\mid z / r_{0}=0.5\right)$

\begin{tabular}{|c|c|c|c|}
\hline $\mathrm{L}_{x} / \mathrm{r}_{0}$ & $\mathrm{~L}_{z} / \mathrm{r}_{0}$ & $\mathrm{~N}$ & $\left|\mathrm{U} 1_{z}\right|(\mathrm{m})$ \\
\hline 4.0 & 8 & 170 & $2.2310^{-8}$ \\
\hline 4.8 & 8 & 192 & $2.7210^{-8}$ \\
\hline 4.0 & 10 & 200 & $2.5710^{-8}$ \\
\hline 4.0 & 12 & 240 & $2.7810^{-8}$ \\
\hline
\end{tabular}

$\mathrm{N}$ : nombre d'éléments du maillage.

Tableau 2. - Déplacement vertical calculé fonction de la taille $\mathrm{lz}$ du maillage $\left(L x / r_{0}=4.8\right.$ et $L z / r_{0}=8$ et $\mid x=0.4$ )

Table 2. - Computed vertical displacement in terms of $\mathrm{Iz}$ extent $\left(\mathrm{Lx} / \mathrm{r}_{0}=4.8, \mathrm{Lz} / \mathrm{r}_{0}=8\right.$ and $\left.\mathrm{I} x=0.4\right)$

\begin{tabular}{|c|c|c|c|}
\hline$I_{z} / r_{0}$ & $N$ & $\left|U 1_{z}\right|(m)$ & $\Delta U / U$ \\
\hline 1.0 & 96 & $3.6710^{-8}$ & $23 \%$ \\
\hline 0.5 & 192 & $2.7210^{-8}$ & $7 \%$ \\
\hline 0.4 & 240 & $2.9210^{-8}$ & - \\
\hline
\end{tabular}

$\mathrm{N}$ : nombre d'éléments du maillage.

pas l'interprétation de l'essai décrit § 2 car la distorsion réalisée expérimentalement sort du cadre de l'hypothèse (4.1.).

La prise en compte d'un nombre suffisant de termes dans le développement de exp $\left(-\alpha_{1}\left|\mathrm{I}_{2}\right|^{1 / 2}\right)$ doit permettre l'interprétation quantitative de cet essai.

La méthode d'interprétation de l'essai S.H.S. ainsi mise au point pourra ensuite être étendue à d'autres configurations en particulier à des essais harmoniques Crosshole effectués en forage, en cours de réalisation sur un principe similaire à lessai de pompage harmonique

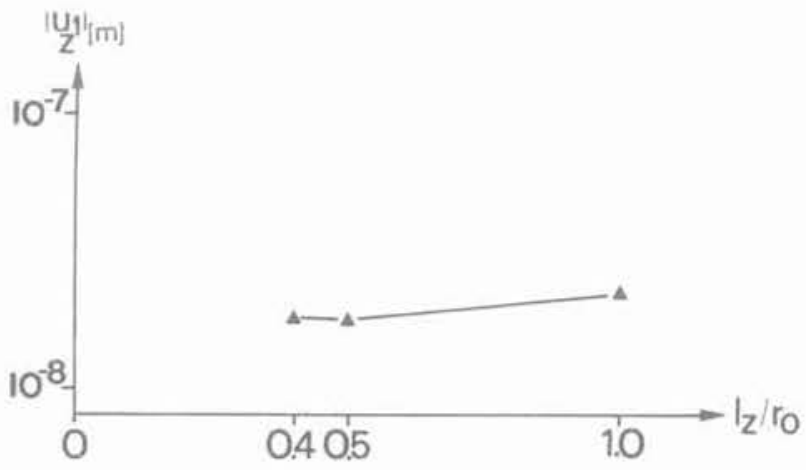

Fig. 10. - Stabilité du processus de calcul: variation du déplacement vertical calculé en fonction de la taille de la maille.

Stability of computation scheme: variation of computed vertical displacement in terms of element size. 


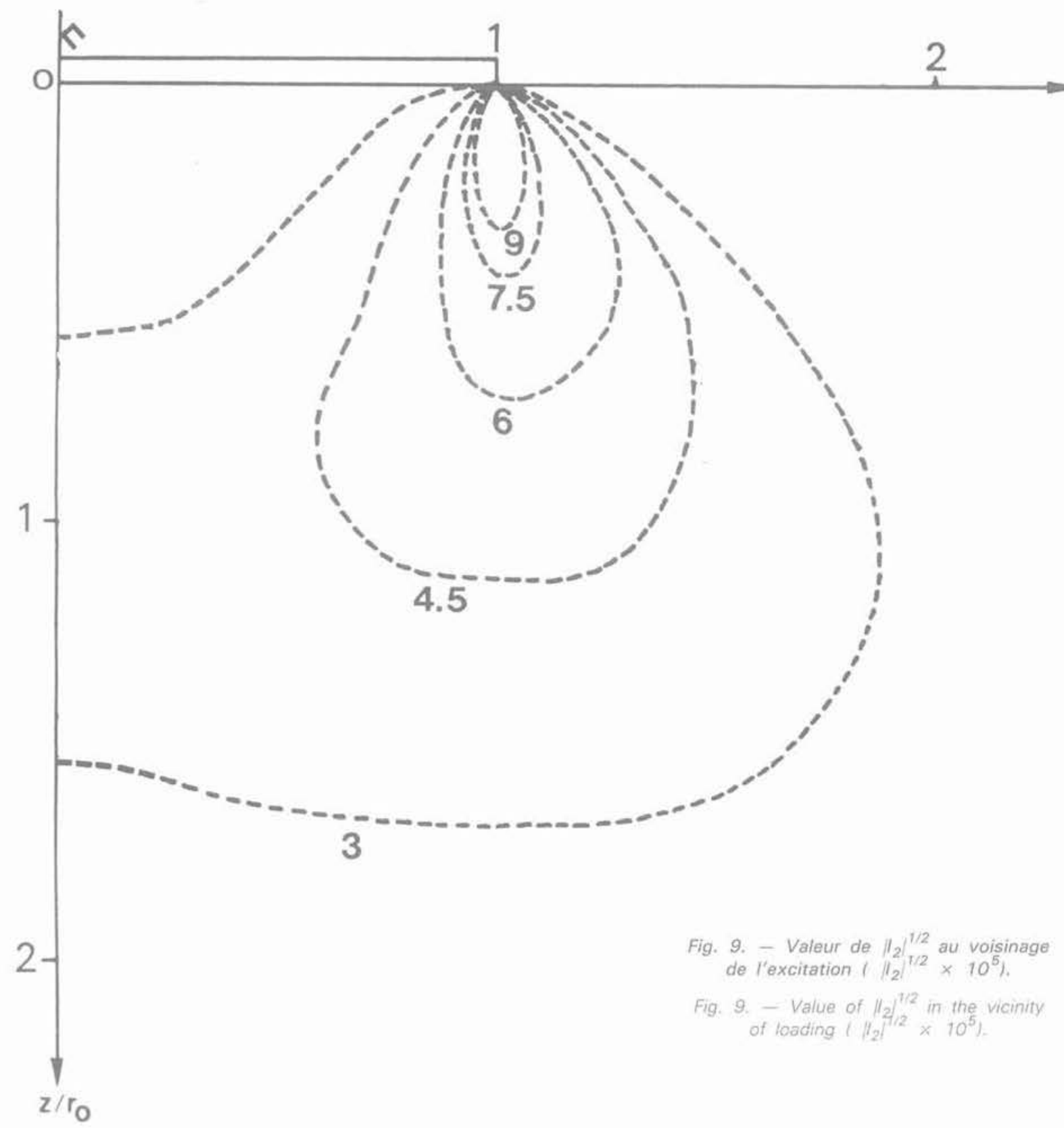

CROSNIER et al. (1983). On aboutira ainsi à une méthode expérimentale permettant d'obtenir les paramètres du comportement non linéaire à l'aide d'essais nécessitant une énergie modérée.

\section{BIBLIOGRAPHIE}

BLAQUIERE A. (1966), Analyse des systèmes non linéaires. Bibliothèque des Sciences et Techniques Nucléaires, I.N.S.T.N, (Saclay) P.U.F,, 1966, p. 88-94.

BREBBIA C.A., TELLES J.C.F., WROBEL L.C. (1984), Boundary Element Techniques. Theory and
Applications in Engineering. SpringerVerlag., Berlin, 1974.

CROSNIER B., PORTALES J.L., FRAS G., JOUANNA P. (1983), Reconnaissance par pompage harmoni. que de milieux rocheux. Matériaux et Constructions vol. $16, n^{\circ} 92$, pp. 71-83, 1983.

GERRARD C.M., HARRISON W.J. (1974), Circular loads applied to a cross-anisotropic half-space in Elastic solutions for Soil and Rock Mechanics (DAVID G. \& POULOS E.), John Wiley and Sons Inc., 1974.

HARDINS B.O., DRNEVICH V.P. (1970), Shear modulus and damping in soils 1 . Measurement and parameter effects, II. Design equations and curves. Techn. Rep. Univ. Kentaky UKY, n०26 and 27-70-CE2. Soil Mechanics series, 1970. 
MANG H.A., HONG-BAO L.I., GUO-MING HAN (1985), A new method for evaluating singular inte. grals in stress analysis of solids by the direct boundary element technique. Int. J. for num. mech. in Eng., vol. 21, 1985, p. 2071-2098.

RICHART F.E., WOODS R.D. (1970), Vibration of Soils and Foundations. Prentice Hall Inc. Englewood Cliffs, New Jersey, 1970, p. 111-120.

RICHART F.E., WYLIE E.B. (1975), Influence of Dynamic soil properties on response soil masses. Symp. Struct. and Geotech. Mech., Un. of Ill, Urbana, Oct., 1975.

SEED H.B., LEE K.L., IDRISS 1.M., MAKDISIF F. (1973), Analyses of the slides in the San Francisco Dams during the Earthquake of Feb. 9 1971. EERC Rep. $n^{\circ} 73-2$, Univ. Of California, Berkeley, 1973.

SHANNON, WILSON, AGABIAN (1980), Evaluation of in situ damping characteristics. NUREG/CR - 1938, sept. $1980,170 \mathrm{p}$.

\section{ANNEXE \\ Composantes du tenseur des déformations en représentation axisymétrique et en approximation linéaire élastique}

Le calcul nécessite de connaître les expressions analystiques des composantes $e_{r r}, e_{z}$. $e_{\theta \theta}$ et $e_{r 2}$ du tenseur des déformations associées au chargement circulaire uniforme $\mathrm{T}$ appliqué en surface d'un demi-espace. Chacune des coordonnées $r$ et $z$, apparaissant dans les expressions qui suivent, est normalisée par $r_{0}$, rayon de charge.

Soit $A=f / 2 \pi r_{0}^{2}$ alors

$$
\begin{aligned}
& e_{\pi}=A\left\{\left[2\left(\lambda_{0}+G_{0}\right)\right]^{-1} \cdot\left(f_{1}^{-1} \sin \frac{f_{2}}{2}-\left(1-f_{1} \sin \frac{f_{2}}{2}\right)\right)\right. \\
& -\left(Z / 2 G_{0}\right) \cdot\left(f_{1}^{-3}\left(Z \sin \frac{3 F_{2}}{2}-\cos \frac{3 F_{2}}{2}\right)\right. \\
& \left.\left.-F_{1}^{-1}\left(\cos \frac{F_{2}}{2}-Z \sin \frac{F_{2}}{2}\right)\right)\right\} \\
& e_{\theta \theta}=A\left\{\left[2\left(\lambda_{0}+G_{0}\right)\right]^{-1} \cdot\left(1-f_{1} \sin \frac{F_{2}}{2}\right)\right. \\
& \left.-\left(Z / 2 G_{0}\right) F_{1}^{-1}\left(\cos \frac{f_{2}}{2}-Z \sin \frac{F_{2}}{2}\right)\right\} \\
& e_{z}=A\left\{[ 2 ( \lambda _ { 0 } + G _ { 0 } ) ] ^ { - 1 } \cdot \left(F_{1}^{-1} \sin \frac{F_{2}}{2}+\left(Z / 2 G_{0}\right) f_{1}^{-3}\right.\right. \\
& \left.\left(Z \sin \frac{3 F_{2}}{2}-\cos \frac{3 F_{2}}{2}\right)\right\} \\
& e_{r z}=\frac{A}{G_{0}} r z f_{1}^{-3} \sin \frac{3 f_{2}}{2}
\end{aligned}
$$

avec :

$$
\begin{aligned}
& f_{1}=\left[\left(z^{2}+r^{2}-1\right)^{2}+4 z^{2}\right]^{1 / 4} \\
& f_{2}=\operatorname{Arctg}\left[2 Z \cdot\left(Z^{2}+r^{2}-1\right)^{-1}\right]
\end{aligned}
$$

